\title{
Virtual Experimental Project Design in the Digital Signal Processing Course
}

\author{
Boyu Si*, Baodan Bai, Lijun Hao, Xiaoou Li \\ School of Medical Instrument, Shanghai University of Medicine \& Health Sciences, Shanghai 201318, China \\ *Corresponding author: Boyu Si, hrbsby@163.com
}

\begin{abstract}
This paper proposes the design and development of virtual experimental projects in the Digital Signal Processing course, using MATLAB, Proteus, and CCS platforms to develop a library of typical experimental cases for biomedical engineering majors and discusses the design process. Based on these typical cases, this paper explores the secondary design for innovative engineering practice case teaching, which can promote students' understanding and mastery of digital signal processing theories, algorithms, and technologies in an intuitive, flexible, and efficient way; quickly build new innovative engineering case models and further cultivate students' engineering application ability as well as innovative thinking.
\end{abstract}

Keywords: Digital Signal Processing course; Virtual experiment; Circuit simulation

Publication date: November 2021; Online publication: November 30, 2021

\section{Introduction}

Digital Signal Processing is an essential course in the undergraduate curriculum of biomedical engineering. It is of great significance to cultivating high-quality medical device engineering talents ${ }^{[1]}$. The theoretical knowledge of this course is complex, and a considerable amount of practice is required for students to understand the technology and methods in signal processing ${ }^{[2]}$. Conventional experimental teaching in Digital Signal Processing is often subjected to equipment, space, teachers, and other objective conditions. The experiments require the use of high-performance computers, digital signal processing experimental boxes, emulators, and other electronic components; in addition, the purchase and maintenance costs are also high ${ }^{[3]}$. At the same time, there are problems such as low teacher-student comparison as well as insufficient laboratory hours and space, which ensue a difficulty in providing students with sufficient practice time, resulting in poor theoretical foundation among students and their inability to really master digital signal processing technology.

Virtual experiment projects may help solve these problems ${ }^{[4,5]}$. Virtual simulation provides learners a complete interactive learning environment through visual, auditory, tactile, and other sensory experiences while carrying out experimental operations, thus helping them to understand the purpose of the experiments and master the main points of the operations. This is due to the fact that virtual experiment projects are often based on the case teaching method, which guides students to flexibly apply the theoretical knowledge that they have learned, think about various problems encountered during the experiments, obtain solutions to problems, and accomplish the experimental objectives, thus enhancing their thinking skills and engineering practical skills through the selection of typical and vivid practical cases. Therefore, this paper proposes a case-based virtual experiment project based on Proteus and MATLAB platforms as well as forms a complete set of virtual experiment library. By completing a series of experimental projects, students' learning interest and innovative thinking can be enhanced and cultivated, respectively. 


\section{Virtual experimental project design}

The design principles of virtual experimental projects include typicality, practicability, frontier, and extensibility. Typicality refers to the requirement of the experimental content to include the classical knowledge and technology in the field of biomedical engineering. Practicability refers to the requirement of cultivating students' practical skills and problem-solving skills. The contents of the virtual experiment library established for biomedical engineering students in the Digital Signal Processing course are shown in Figure 1. Frontier refers to the requirement of experimental topics to present the most cutting-edge research results in the field of biomedical signal processing. Extensibility refers to the requirement of the experimental program to support secondary development and utilization, saving design time.

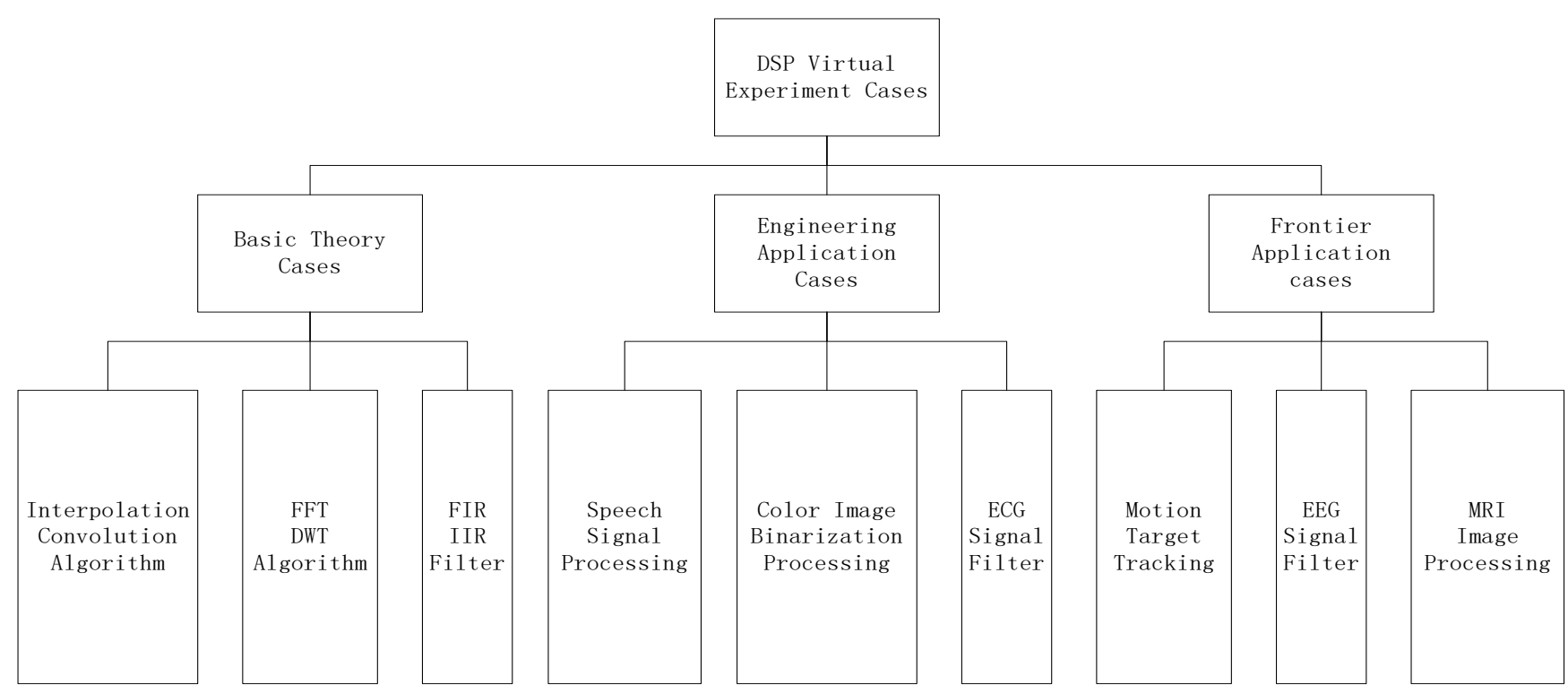

Figure 1. A virtual experiment library for the Digital Signal Processing course

\section{Virtual experiment platform selection}

MATLAB, Proteus, and CCS (Code Composer Studio) are used to build typical case models, such as filter design, fast Fourier transform (FFT), wavelet decomposition, etc., which can help students quickly design and verify the suitability of signal processing algorithms. Proteus is a classic simulation circuit design software, supporting rich virtual components, used to build digital signal processor (DSP) chips and peripheral circuits, supporting assembly programs. CCS is used to develop experimental programs for DSP chips and can be jointly debugged with Proteus to realize hardware simulation.

\section{Development process of virtual experimental projects}

The basic development process of the experimental project is as follows: (1) determine the digital signal processing algorithm used according to the experimental requirements, write $\mathrm{M}$ programs in MATLAB, build mathematical models, and conduct tests; (2) write C programs in CCS or a mixture of C and assembly language programming as well as compile to generate COF files; (3) design and complete the simulation experimental circuit in Proteus, import the COF file in Proteus, debug, and run.

Through the above experimental steps, an all-round training for students can be realized from conception, theoretical analysis, programming, to hardware design. 


\section{Typical virtual experiment case: FIR filter design}

The design and development of a virtual experiment project is illustrated by the example of FIR filter design, which is an important element in the Digital Signal Processing course. FIR filter is a finite impulse response filter, whose output depends only on the past input but independent of the past output, and whose unit impulse response is a finite term. Strict linear phase can be achieved when certain symmetry conditions are satisfied. In the fields of data transmission, image processing, and pattern recognition, it is required that the signal should not have significant phase distortion during processing, so the linear phase FIR filter is widely used. In this paper, the steps in developing the FIR filter design virtual experiment are discussed.

\subsection{Principle analysis and MATLAB modeling}

In the design of FIR filter, students first need to master the technical principles of digital filtering of analog signals. The design methods mainly include the window function method and the frequency sampling method. The design indexes include sampling frequency, cutoff frequency, attenuation coefficient, etc. Students can use the FDATool toolbox in the MATLAB platform for filter modeling to compare, select, and apply the above indicators for better understanding of the theoretical knowledge of FIR filters. For the experiment, on the running interface of the FDATool toolbox, the high-pass filter and window function method are selected to design the FIR filter. The design specifications are set as follows: input sampling frequency, Fs $=8 \mathrm{kHz}$; passband cutoff frequency, $\mathrm{Fp}=1.05 \mathrm{kHz}$; stopband cutoff frequency, Fst $=1 \mathrm{kHz}$; passband maximum attenuation, $A p=1 \mathrm{~dB}$; stopband minimum attenuation $A s t=80 \mathrm{~dB}$. The spectral curve of the filter function can then be obtained to clarify the relationship between the design index and the filter performance.

\subsection{CCS generates COF files}

CCS is an integrated DSP software development tool that supports Texas Instruments (TI)'s family of microcontrollers and embedded processors to help developers write, compile, and debug development code for DSP chips. For experiments, the set 16-bit signed integer filter coefficients can be obtained through the integrated development environment (IDE) function of CCS and saved in the form of an array of C programs. The array elements exhibit even-symmetric characteristics and satisfy the linear phase characteristics of the FIR filter. Since CCS does not support direct on-line debugging with Proteus, CCS is used to generate COF files for loading onto the DSP chip used to build the filter circuit using Proteus.

\subsection{Proteus-designed digital filter circuit}

The digital filter circuit consists of a pre-processing circuit, an analog to digital (A/D) conversion sampling circuit, a DSP filter circuit, a D/A conversion circuit, and an output smoothing circuit. The initial analog signal is first pre-processed by limiting and using an anti-aliasing filter to meet the sampling requirements, and after A/D conversion and sampling, it is input to the DSP filter circuit for filtering operation; the result is generated by the D/A converter circuit to generate the analog signal, and finally the filtered signal is output by the smoothing circuit. The schematic diagram of the design circuit in Proteus is shown in Figure 2. Two analog signals are superimposed as the input signal, and TMS320F28027 is selected as the main DSP chip to realize A/D conversion and filtering. Two DAC0832 chips are used to complete the D/A conversion and output the analog signal. The simulation circuit is run for debugging. The virtual oscilloscope observed that the waveforms before and after filtering are as expected. 


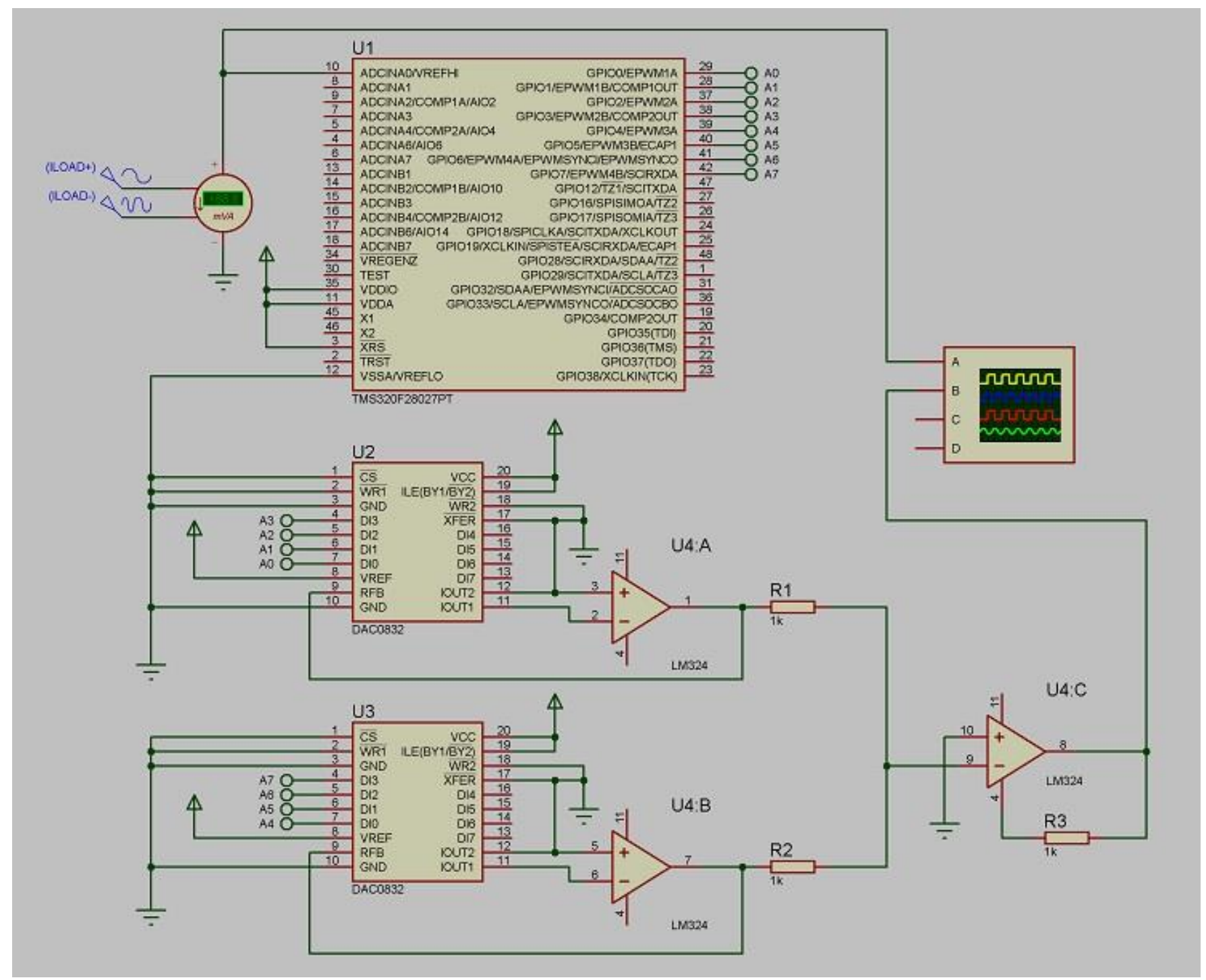

Figure 2. Simulation of a filter circuit

\section{Secondary development of virtual experimental projects}

Besides the classical filtering role, there are many application scenarios of FIR filters in the field of biomedical engineering. With the help of FIR filter cases in the virtual lab library, new engineering project development can be realized, which is the secondary development of FIR filter application cases. The application of FIR filters in speech signal acquisition and processing system is illustrated below as a secondary development case. Speech signal acquisition and processing system is widely used in communication, medical, multimedia, and other fields. The main functions include voice signal acquisition, A/D conversion, filtering, D/A conversion, and spectrum display. The filtering part adopts FIR filter, and the development work mainly includes two aspects: first, the software implementation of FIR algorithm; second, the hardware timing control of the input and output data streams. In the process of designing the FIR filter, according to the human voice signal frequency range of $300 \mathrm{~Hz}$ to $3000 \mathrm{~Hz}$, the filter type is changed to low-pass filter, the cut-off frequency is modified to $3000 \mathrm{~Hz}$, and a frequency of $8000 \mathrm{~Hz}$ is adopted, which can realize the filtering of high-frequency interference and ensure that there is no loss of sound quality in the voice.

In practice, the comparison method can be used to set the cutoff frequencies to $1000 \mathrm{~Hz}, 2000 \mathrm{~Hz}$, and $3000 \mathrm{~Hz}$, respectively and compare the spectrograms before and after filtering, which can be more intuitive 
to view the filtering effect. Meanwhile, by using headphones to monitor the filtering effect, it can be appreciated that when the cutoff frequency is set to $1000 \mathrm{~Hz}$, the processed speech becomes low and choppy, losing a lot of information; when the cutoff frequency is set to $2000 \mathrm{~Hz}$, the processed speech is brighter than the former, but there is still a loss of sound quality; the effects from the two schemes are obviously not as good as the effect when the cutoff frequency is set to $3000 \mathrm{~Hz}$. This experimental result corresponds exactly to the theory that the frequency of human speech is in the range of $300 \mathrm{~Hz}$ to $3400 \mathrm{~Hz}$. As can be seen, the secondary development of FIR filter application can further deepen students' understanding of FIR filter design methods and also improve their practical application skills. Meanwhile, the secondary development of virtual experiment cases overcomes the limitation of experimental conditions and expands the application scope of innovative engineering practice cases.

\section{Conclusion}

In this paper, the development and secondary design of virtual experimental cases in Digital Signal Processing have been discussed, solving the problem of insufficient space and experimental equipment in innovative engineering experimental teaching. Using MATLAB, Proteus, and other platforms, a case library has been designed for biomedical engineering applications; in addition, the secondary development and application of typical cases have been explored, which can realize the training of innovative engineering practice ability of "design - theory - software - hardware" in an intuitive, flexible, and effective way.

\section{Funding}

This study was supported by the 2021 Experimental Teaching Reform Project of Shanghai University of Medicine \& Health Sciences "Digital Signal Processing Course Design" (Project Number: JG(21)04-B402).

\section{Disclosure statement}

The authors declare that there is no conflict of interest.

\section{Author contributions}

Si Boyu and Li Xiaoou conceived the idea of the study. Bai Baodan and Zha Yutong performed the experiments. Si Boyu wrote the paper.

\section{References}

[1] Yang X, Li F, Xue H, et al., 2014, DSP Technology and Application for Electric Power Majors. Exploration of Course Teaching Reform. China Electric Power Education, (30): 45-46. http://10.19429/j.cnki.cn11-3776/g4.2014.30.022

[2] Du P, 2012, The Application of Comparative Teaching Method in the Course of "DSP Technology and Application". China Electric Power Education, (6): 75. http://10.3969/j.issn.1007-0079.2012.06.032

[3] Chen Z, Wang J, 2014, Application of Proteus Simulation Software in Electrical Engineering. Experimental Technology and Management, 31(2): 93-95. http://10.16791/j.cnki.sjg.2014.02.025

[4] Yao R, Li Z, Fu D, et al., 2015, Design and Development of Proteus-Based DSP Virtual Experiment System. Experimental Technology and Management, 32(3): 123-125, 136. http://10.16791/j.cnki.sjg.2015.03.032 
[5] Zhu L, Song C, 2017, Design of DSP-Based FIR Digital Filtering Integrated Experiment. Experimental Technology and Management, 34(1): 206-209, 213. http://10.16791/j.cnki.sjg.2017.01.050 